Supporting Information

\title{
Data-driven prediction of minimum fluidization velocity in gas-fluidized beds using data extracted by text mining
}

\author{
Jibin Zhou ${ }^{1}$, Duiping Liu ${ }^{1}$, Mao Ye ${ }^{1 *}$, Zhongmin Liu ${ }^{1,2}$ \\ 1. National Engineering Laboratory for Methanol to Olefins, Dalian Institute of \\ Chemical Physics, Chinese Academy of Sciences, Dalian 116023, China. \\ 2. University of Chinese Academy of Sciences, Beijing 100083, China. \\ *Corresponding author: maoye@dicp.ac.cn
}


Table S1. The extracted and calculated values of gas density, gas viscosity, pressure and temperature.

\begin{tabular}{cccccccc}
\hline $\boldsymbol{\rho}_{\boldsymbol{g}}$ & $\boldsymbol{\mu}$ & $\mathbf{P}$ & $\mathbf{T}$ & $\boldsymbol{\rho}_{\boldsymbol{g}}^{\prime}$ & $\Delta \boldsymbol{\rho}$ & $\boldsymbol{\mu}^{\prime}$ & $\Delta \boldsymbol{\mu}$ \\
\hline 1.252 & $2.00 \mathrm{E}-05$ & 100 & 423 & 0.831 & 0.336 & $2.40 \mathrm{E}-05$ & 0.198 \\
0.348 & $4.24 \mathrm{E}-05$ & 100 & 1023 & 0.344 & 0.012 & $4.30 \mathrm{E}-05$ & 0.013 \\
0.558 & $3.14 \mathrm{E}-05$ & 100 & 623 & 0.564 & 0.011 & $3.14 \mathrm{E}-05$ & 0.002 \\
0.599 & $3.63 \mathrm{E}-05$ & 100 & 923 & 0.381 & 0.364 & $4.03 \mathrm{E}-05$ & 0.111 \\
1.13 & $1.80 \mathrm{E}-05$ & 100 & 298.15 & 1.179 & 0.043 & $1.84 \mathrm{E}-05$ & 0.022 \\
1.25 & $1.80 \mathrm{E}-05$ & 100 & 298 & 1.180 & 0.056 & $1.84 \mathrm{E}-05$ & 0.021 \\
1.21 & $1.81 \mathrm{E}-05$ & 100 & 298 & 1.180 & 0.025 & $1.84 \mathrm{E}-05$ & 0.016 \\
1.21 & $1.81 \mathrm{E}-05$ & 100 & 298 & 1.180 & 0.025 & $1.84 \mathrm{E}-05$ & 0.016 \\
1.21 & $1.80 \mathrm{E}-05$ & 100 & 293 & 1.200 & 0.008 & $1.81 \mathrm{E}-05$ & 0.008 \\
1.225 & $1.79 \mathrm{E}-05$ & 230 & 647 & 1.249 & 0.020 & $3.21 \mathrm{E}-05$ & 0.796 \\
1.225 & None & 100 & 373 & 0.943 & 0.231 & $2.18 \mathrm{E}-05$ & None \\
1.22 & $1.79 \mathrm{E}-05$ & 100 & 293 & 1.200 & 0.016 & $1.81 \mathrm{E}-05$ & 0.013 \\
\hline None & $4.58 \mathrm{E}-05$ & 100 & 1120 & 0.314 & None & $4.54 \mathrm{E}-05$ & 0.009 \\
1.17 & $1.75 \mathrm{E}-05$ & 100 & 297 & 1.184 & 0.012 & $1.83 \mathrm{E}-05$ & 0.048 \\
1.2 & $1.80 \mathrm{E}-05$ & 100 & 297 & 1.184 & 0.013 & $1.83 \mathrm{E}-05$ & 0.019 \\
1.21 & $1.81 \mathrm{E}-05$ & 100 & 298 & 1.180 & 0.025 & $1.84 \mathrm{E}-05$ & 0.016 \\
0.11 & None & 10 & 298 & 0.118 & 0.073 & $1.84 \mathrm{E}-05$ & None \\
1.12 & None & 100 & 298 & 1.180 & 0.053 & $1.84 \mathrm{E}-05$ & None \\
0.55 & None & 50 & 298 & 0.590 & 0.073 & $1.84 \mathrm{E}-05$ & None \\
0.33 & None & 30 & 298 & 0.354 & 0.073 & $1.84 \mathrm{E}-05$ & None \\
\hline & $1.80 \mathrm{E}-05$ & 100 & 298 & 1.180 & 0.017 & $1.84 \mathrm{E}-05$ & 0.021 \\
\hline
\end{tabular}

$\rho_{g}^{\prime}, \mu^{\prime}$ are the calculated values according to the Eqs 10-11.

$\Delta \rho=\left(\rho_{g}-\rho_{g}^{\prime}\right) / \rho_{g} ; \Delta \mu=\left(\mu-\mu^{\prime}\right) / \mu$ 
Table S2. Results of Ergun and Wen-Yu correlations for different Geldart Groups particles.

\begin{tabular}{lcccccccccc}
\hline \multirow{2}{*}{ Correlation } & \multicolumn{3}{c}{ A } & \multicolumn{4}{c}{ B } & \multicolumn{3}{c}{ D } \\
\cline { 2 - 10 } s & RMSE & MAE & $\mathbf{R}^{2}$ & RMSE & MAE & $\mathbf{R}^{2}$ & RMSE & MAE & $\mathbf{R}^{2}$ \\
\hline Ergun & 1.782 & 0.722 & 0.563 & 11.553 & 6.640 & 0.0044 & 25.777 & 20.544 & 0.610 \\
Wen-Yu & 2.120 & 0.724 & 0.382 & 7.833 & 3.360 & 0.542 & 17.848 & 10.281 & 0.813 \\
\hline
\end{tabular}



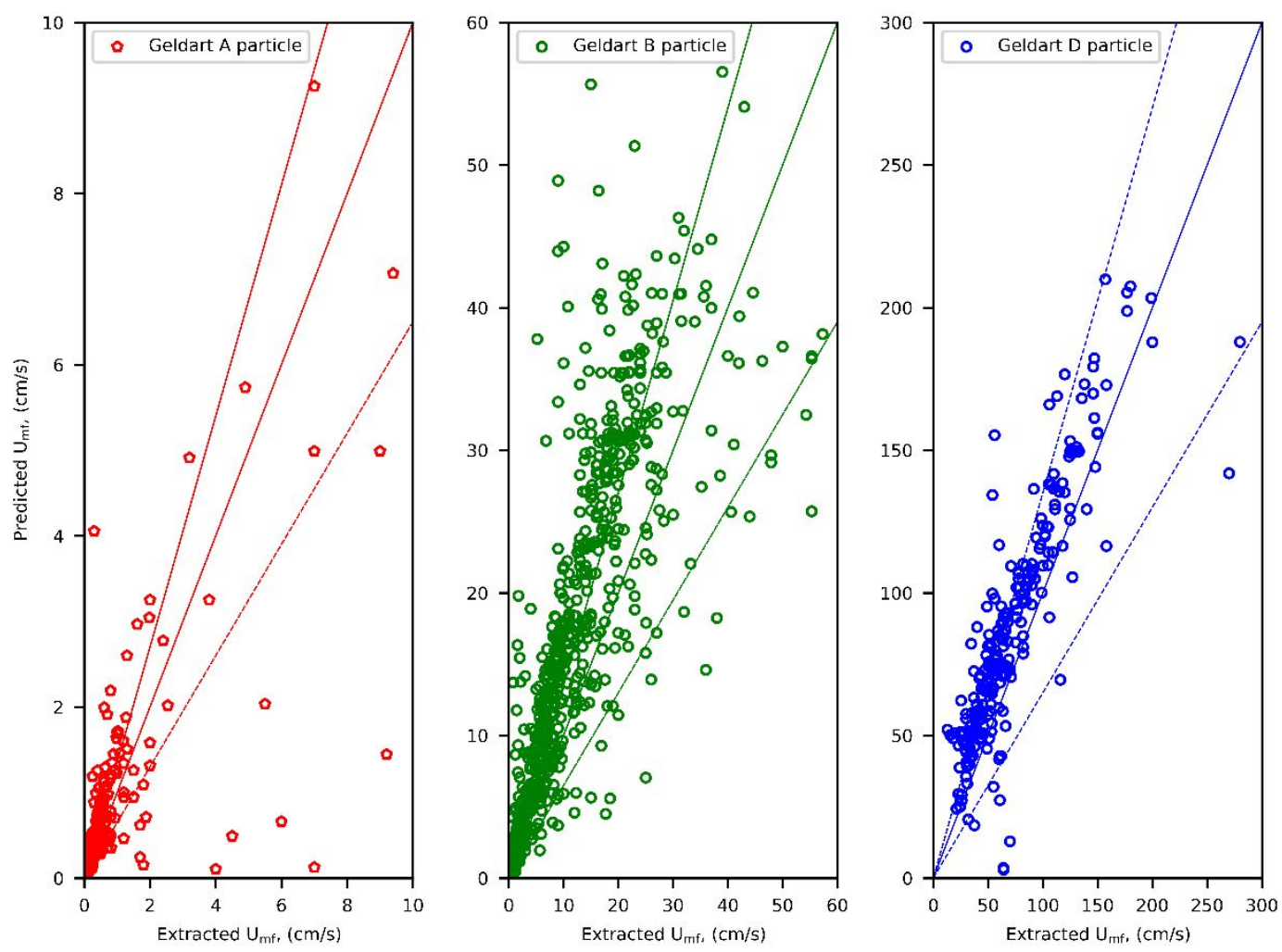

Figure S1. Comparisons of the predicted and extracted values of $U_{m f}$ for Ergun correlation in view of Geldart Group A, B and D particles. 

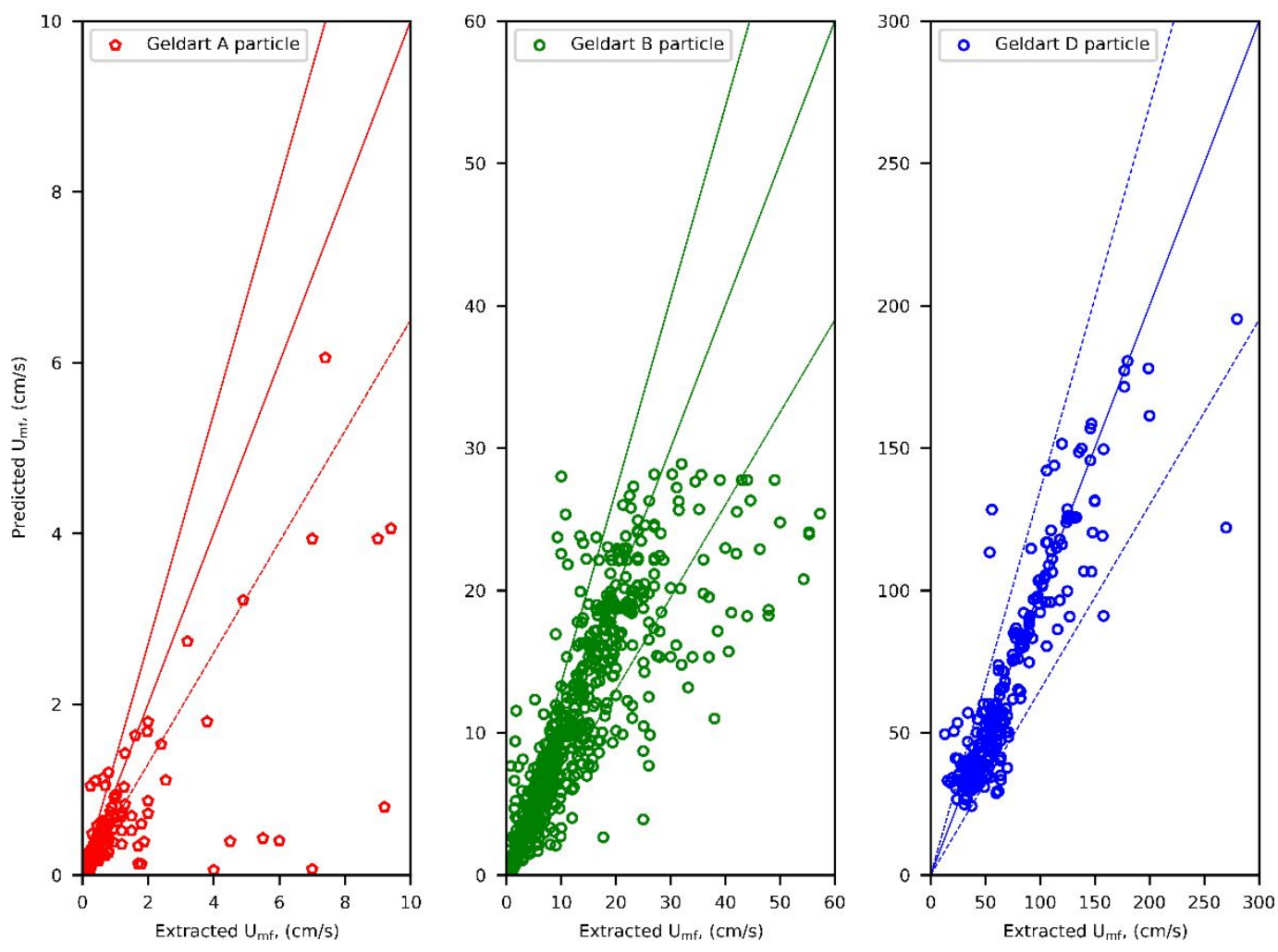

Figure S2. Comparisons of the predicted and extracted values of $U_{m f}$ for Wen-Yu correlation in view of Geldart Group A, B and D particles. 
Table S3. The performances of ANN models with different parameters.

\begin{tabular}{llccc}
\hline Test size & ANN model & RMSE & MAE & $\mathbf{R}^{2}$ \\
\hline \multirow{2}{*}{0.3} & 3 layers (32-16-8) & 8.607 & 2.038 & 0.927 \\
& 2 layers (16-8) & 8.684 & 2.269 & 0.926 \\
& 1 layer and 32 nodes & 9.032 & 2.195 & 0.919 \\
& 1 layer and 64 nodes & 8.256 & 2.292 & 0.933 \\
\hline 0.2 & 1 layer and 16 nodes & 7.989 & 2.181 & 0.937 \\
\hline 0.4 & 1 layer and 16 nodes & 7.683 & 2.277 & 0.932 \\
\hline
\end{tabular}



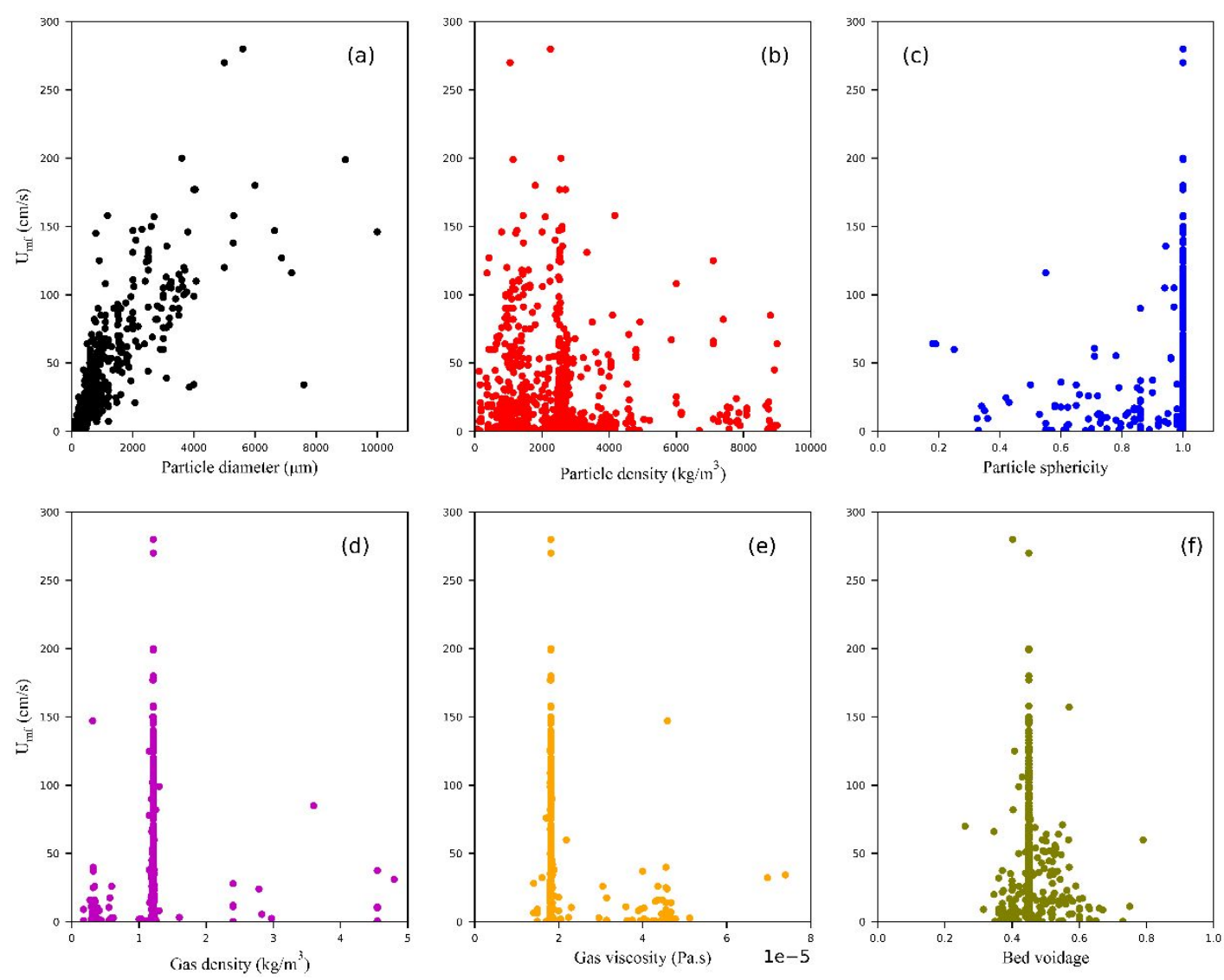

Figure S3. Relationships between the $U_{m f}$ and particle diameter (a), particle density(b), particle sphericity (c), gas density (d), gas viscosity (e) and bed voidage (f). 


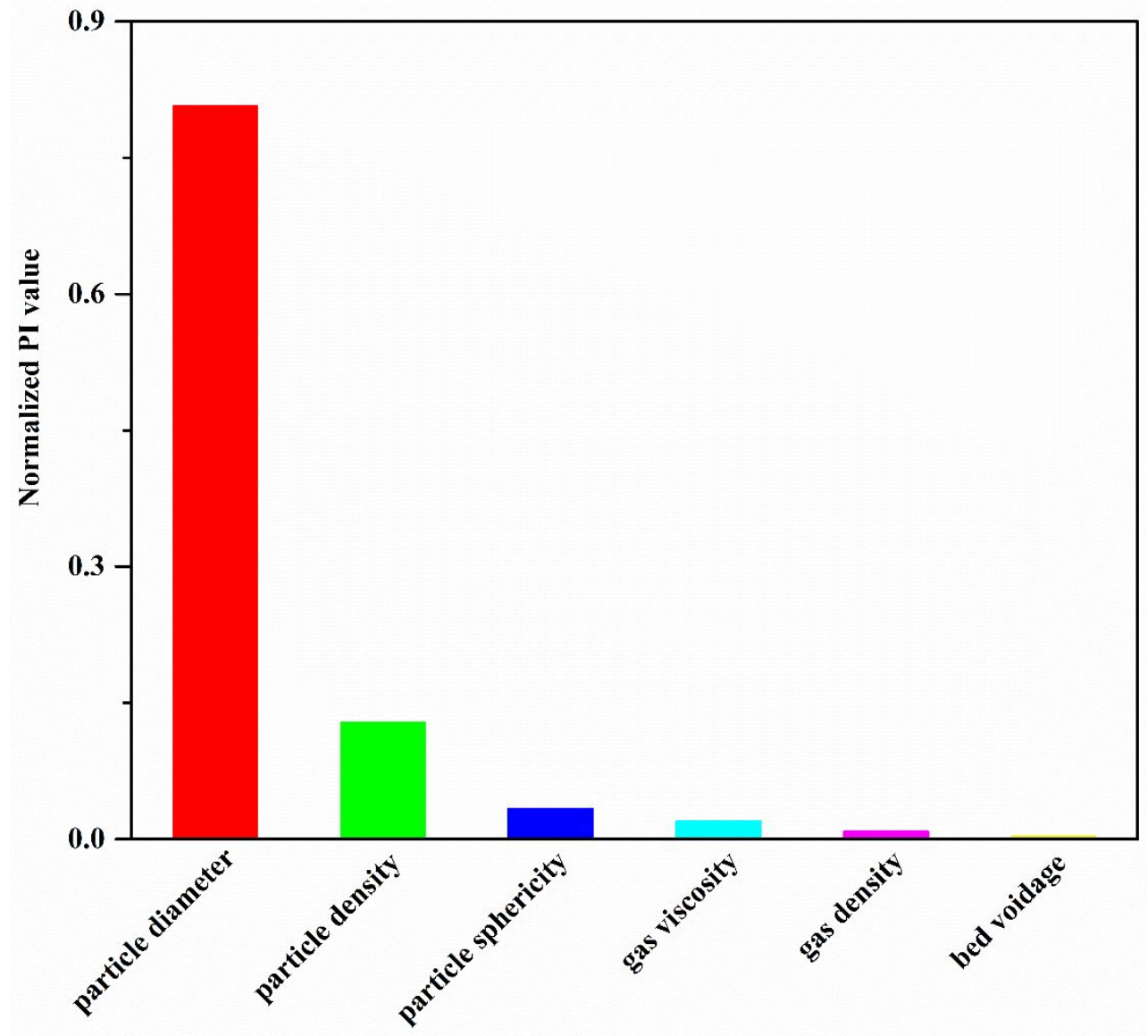

Figure S4. The normalized PI value of the features. 